Check for updates

Cite this: RSC Adv., 2017, 7, 49969

\title{
Rapid detection of hexamethylenetetramine based on the substrate UC@SiO 2 @AuaAg using SERS
}

\author{
Rui Tong, ${ }^{a}$ Xiaopeng Hu, ${ }^{a}$ Guozhen Fang, (D) *a Shiming Sun, ${ }^{a}$ Jingmin Liu ${ }^{b}$ \\ and Shuo Wang ${ }^{\star a c}$
}

A reliable upconversion (UC) material assembled from Au@Ag (UC@SiO $2 @ A u @ A g)$, as the substrate, was firstly employed to detect hexamethylenetetramine (HMT) in bean vermicelli by surface enhanced Raman spectroscopy (SERS). In this substrate, UC can convert near-infrared (NIR) light into visible light to promote localized surface plasmon resonance (LSPR) of noble metal nanoparticles to enhance the SERS signal. According to optimized synthesis conditions, the SERS substrate was obtained and evaluated. The substrate shows uniformity with RSD of $7.05 \%$ and $7.37 \%$ for the Raman vibrations at $\sim 780 \mathrm{~cm}^{-1}$ and $\sim 1043 \mathrm{~cm}^{-1}$ respectively, and sensitivity with detection limits of $1 \mathrm{mg} \mathrm{L}^{-1}$ for HMT. Based on the better sensitivity of this method, the artificially positive samples have been detected.

Received 11th July 2017

Accepted 18th October 2017

DOI: $10.1039 / \mathrm{c} 7 \mathrm{ra07634g}$

rsc.li/rsc-advances

with tandem mass spectrometry (HPLC-MS/MS). ${ }^{4}$ Although these methods are accurate, reliable and sensitive, they are time consuming, expensive and labour-intensive. ${ }^{5}$ Therefore, an advanced method that is rapid, has a simple operation and is inexpensive is crucial to detect HMT in food products.

Surface enhanced Raman spectroscopy (SERS), as an important spectroscopic analytical method, with rapidness and simple operation has been widely used in chemical detections because of their unique spectral fingerprints. The signal of SERS is much higher than that of normal Raman, which mainly due to a combination of electromagnetic and chemical mechanisms. ${ }^{6-10}$ Strong electromagnetic fields are generated when the LSPR of metal nanoparticles is excited by visible light. So the Raman signal will be enhanced. Fortunately, because of multiple photon absorptions or energy transfer, ${ }^{11,12} \mathrm{NaYF}_{4}, \mathrm{Yb}$, Er upconversion (UC) materials can convert near-infrared (NIR) light into visible light. Besides, UC materials have the advantages of low toxicity and chemical stability ${ }^{13}$ Thus, UC materials assembling noble metal nanoparticles can generate good SERS performance under NIR laser excitation. ${ }^{14,15}$

In this work, UC materials assembled Au@Ag (UC@SiO ${ }_{2} @-$ $\mathrm{Au} @ A g)$ was firstly employed as SERS substrate to detect HMT in the artificially positive samples. Schematic representation of synthesis of $\mathrm{UC} @ \mathrm{SiO}_{2} @ \mathrm{Au} @ \mathrm{Ag}$ and detection of HMT was showed in Fig. 1.

\section{Experimental}

${ }^{a}$ Key Laboratory of Food Nutrition and Safety, Ministry of Education, Tianjin University of Science and Technology, Tianjin 300457, China. E-mail: fangguozhen@tust.edu.cn; s.wang@tust.edu.cn

${ }^{b}$ Research Center of Food Science and Human Health, School of Medicine, Nankai University, Tianjin 300071, China

${ }^{c}$ Beijing Advanced Innovation Centre for Food Nutrition and Human Health, Beijing Technology \& Business University, Beijing 100048, China

\subsection{Chemicals and materials}

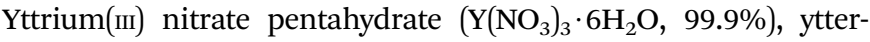
bium(III) nitrate pentahydrate $\left(\mathrm{Yb}\left(\mathrm{NO}_{3}\right)_{3} \cdot 6 \mathrm{H}_{2} \mathrm{O}, 99.9 \%\right)$, erbium(III) nitrate pentahydrate $\left(\mathrm{Er}\left(\mathrm{NO}_{3}\right)_{3} \cdot 6 \mathrm{H}_{2} \mathrm{O}, 99.9 \%\right)$, L-ascorbic acid ( $\geq 99.0 \%), 3$-aminopropyltrimethoxysilane (APTES), 
tetraethyl orthosilicate (TEOS), gold(III) chloride trihydrate $\left(\mathrm{HAuCl}_{4} \cdot 3 \mathrm{H}_{2} \mathrm{O}\right)$, L-ascorbic acid were obtained from Sigma (Sigma-Aldrich, USA), silver nitrate $\left(\mathrm{AgNO}_{3}\right)$ and trisodium citrate were obtained from Sinopharm Chemical Reagent Co., Ltd (Beijing, China). Other reagents were all obtained from Sinopharm Chemical Reagent Co., Ltd (Beijing, China). Urotropine was purchased from Dr Ehrenstorfer GmbH. Bean vermicelli was purchased from the local supermarket. Ultrapure water ( $\geq 18 \mathrm{M} \Omega$, Milli-Q, Millipore) was used throughout the experiment.

\subsection{Instruments}

UV-vis absorption spectroscopy was used to determine the LSPR absorption of the Au@Ag nanoparticles (Thermo Scientific Evolution $300 \mathrm{UV}$-vis Spectrometer). Analysis of the UC@ $\mathrm{SiO}_{2}$ was performed using transmission electron microscopy (TEM, JEOL-2100F). Analysis of morphology of UC were recorded using scanning electron microscope (SEM, SU-8010). The Raman spectra were recorded with a Raman spectrometer (Renishaw Inc., Gloucestershire, UK) equipped with a $785 \mathrm{~nm}$ laser, a Renishaw CCD camera and a 50 objective lens. A HPLC system (Shimadzu, Tokyo, Japan) consisting of a Shimadzu UV detector was used to confirm the results.

\subsection{Synthesis of Au@Ag nanoparticles}

The AuNPs of $10 \mathrm{~nm}$ were used as seeds to prepare Au@Ag nanoparticles via the modified method. ${ }^{16-19}$ Firstly, trisodium citrate $(3 \mathrm{~mL}, 1 \%)$ was rapidly injected into boiling water (99 mL) with $1 \mathrm{~mL} \mathrm{HAuCl} \cdot \cdot 3 \mathrm{H}_{2} \mathrm{O}(1 \%)$ aqueous solution. After the mixture was kept boiling for $30 \mathrm{~min}$, cooled down in room temperature and stored at $4{ }^{\circ} \mathrm{C}$. Secondly, $2 \mathrm{~mL}$ of $0.1 \mathrm{~mol} \mathrm{~L}^{-1}$ L-ascorbic acid was put into $10 \mathrm{~mL}$ AuNPs at stirring for $5 \mathrm{~min}$. Then $\mathrm{AgNO}_{3}$ aqueous solution was added drop by drop into the above solution and the mixture continued to stir for $60 \mathrm{~min}$. After that, the obtained products were centrifuged for $10 \mathrm{~min}$ at $10000 \mathrm{rpm}$, then re-dispersed in $10 \mathrm{~mL}$ ultrapure water and stored at $4{ }^{\circ} \mathrm{C}$.

\subsection{Synthesis of upconversion microcrystals material}

UC microcrystals were prepared by a modified typical hydrothermal method. ${ }^{\mathbf{1 1 , 2 0 , 2 1}}$ In briefly, $\mathrm{NaOH}(0.7 \mathrm{~g})$, oleic acid $(8 \mathrm{~mL})$ and ethanol $(8 \mathrm{~mL})$ were mixed under vigorously stirring. Then $5 \mathrm{~mL}$ ultrapure water, $3 \mathrm{~mL}\left(0.101 \mathrm{~g} \mathrm{~mL}^{-1}\right) \mathrm{NaF}$ solution and $1 \mathrm{mmol}$ (total amount) of aqueous solution of $\mathrm{Y}\left(\mathrm{NO}_{3}\right)_{3} \cdot 6 \mathrm{H}_{2} \mathrm{O}$ (1.56 mL), $\mathrm{Yb}\left(\mathrm{NO}_{3}\right)_{3} \cdot 6 \mathrm{H}_{2} \mathrm{O}(400 \mu \mathrm{L})$ and $\operatorname{Er}\left(\mathrm{NO}_{3}\right)_{3} \cdot 6 \mathrm{H}_{2} \mathrm{O}(40 \mu \mathrm{L})$ (lanthanide ion molar, $\mathrm{Y} / \mathrm{Yb} / \mathrm{Er}=78 / 20 / 2$ ) were sequentially added to the above solution under magnetic stirring. Finally the mixture was transferred to a $50 \mathrm{~mL}$ autoclave and heated at $230{ }^{\circ} \mathrm{C}$ for $12 \mathrm{~h}$. The products were separated by centrifugation and washed 3 times with ethanol, then dried overnight in a vacuum oven at $60{ }^{\circ} \mathrm{C}$.

\subsection{Synthesis of amino-modified UC@SiO}

To make the above UC microcrystals easily bond with Au@Ag nanoparticles, amine functionalization of the microcrystals surface was further prepared. ${ }^{22}$ Typically, $50 \mathrm{mg}$ UC materials was dispersed in a solution containing ethanol $(80 \mathrm{~mL})$ and ultrapure water $(20 \mathrm{~mL})$ by sonication for $30 \mathrm{~min}$. Then $2 \mathrm{~mL}$ of $28 \%$ ammonia solution and TEOS were added to the mixture, respectively. The mixture continuously stirred for $8 \mathrm{~h}$. Subsequently, $200 \mu \mathrm{L}$ of APTMS was added dropwise and continued to react for another $1 \mathrm{~h}$. After that, the products were centrifuged and rinsed 3 times with ultrapure water and ethanol, and then vacuum dried at $60{ }^{\circ} \mathrm{C}$ for $12 \mathrm{~h}$.

\subsection{Synthesis of UC@SiO}

To synthesis of UC@SiO ${ }_{2} @ A u @ A g$ hybrid composites, Au@Ag nanoparticles was swiftly added to $1 \mathrm{~mL}$ amine modified UC@ $\mathrm{SiO}_{2}$ microcrystals $\left(1 \mathrm{mg} \mathrm{mL} \mathrm{mL}^{-1}\right)$. The mixture was slowly rotated for $12 \mathrm{~h}$. Finally, the products were washed 3 times with ultrapure water, collected after centrifugation, and re-dispersed into $1 \mathrm{~mL}$ ultrapure water.

\subsection{Sample preparation}

The HMT standard solutions $\left(20,50,80 \mathrm{mg} \mathrm{L}^{-1}\right)$ were prepared. And the real samples (5 g) were soaked in $100 \mathrm{~mL}$ different concentrations standard solutions overnight and air-dry. Then the artificially positive samples were extracted using $10 \mathrm{~mL}$ acetonitrile by sonication for $30 \mathrm{~min}$. The solution was transferred to another tube to dry by flushing with nitrogen. And then the residue was reconstituted with $0.5 \mathrm{~mL}$ of acetonitrile.

\section{Results and discussion}

\subsection{Synthesis of UC@SiO}

UC@SiO ${ }_{2} @ A u @ A g$ was synthetized by assembling. In order to obtain the best Raman enhancement effect, some synthetic conditions of UC@SiO ${ }_{2} @ A u @ A g$ need to be optimized. These include the thickness of $\mathrm{SiO}_{2}$ layer on $\mathrm{UC}$, the thickness of $\mathrm{Ag}$ shell and the amount of Au@Ag NPs.

To test the effect of $\mathrm{SiO}_{2}$ thickness on the Raman signal, the volumes of TEOS were set to $0,20,40,60,100 \mu \mathrm{L}$ to obtain different silica thicknesses, respectively. As is shown in Fig. 2, when increasing volume of TEOS, the Raman signal intensity was obviously increased with the amount from $0 \mu \mathrm{L}$ to $40 \mu \mathrm{L}$.

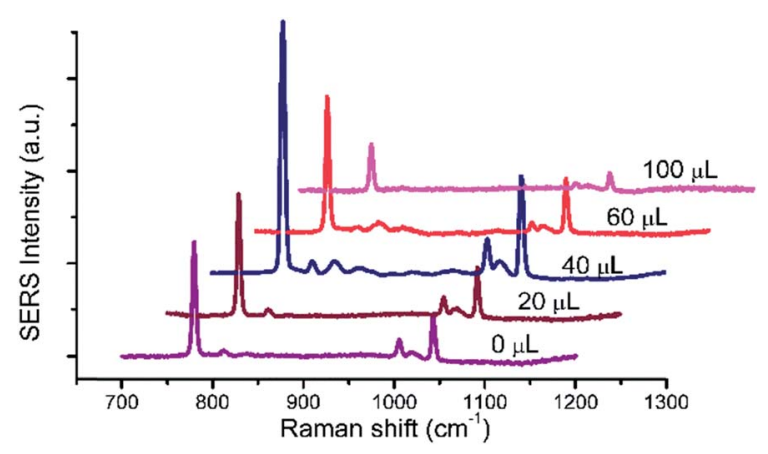

Fig. 2 The Raman spectra of $1 \times 10^{3} \mathrm{mg} \mathrm{L}^{-1} \mathrm{HMT}$ with different

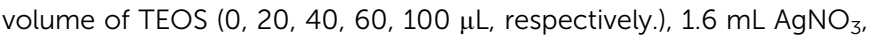
$100 \mu \mathrm{L}$ Au@Ag NPs. 


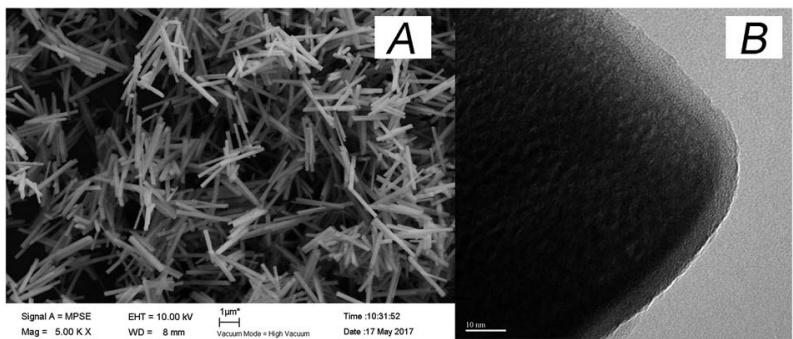

Fig. 3 (A) SEM image of UC; (B) TEM image of $\mathrm{UCASiO}_{2}$ with $40 \mu \mathrm{L}$ TEOS.

This result may be for existing silicon shell to prevent phase change of UC. When the amount of TEOS over $40 \mu \mathrm{L}$ the Raman signal was gradually reduced. Because too thickness of silicon shell brought about that incident light cannot be effective absorbed by UC microcrystals. The SEM image (Fig. 3A) reveals the uniform stick UC microcrystal with diameters about $200 \mathrm{~nm}$ and lengths about $2 \mu \mathrm{m}$, the TEM image (Fig. 3B) shows that $\mathrm{SiO}_{2}$ layer were uniformly coated in UC microcrystal with $40 \mu \mathrm{L}$ TEOS. Thus, $40 \mu \mathrm{L}$ TEOS was selected in the following experiments.

The thickness of Ag shell was also an important factor for Raman intensity. With the volume of $\mathrm{AgNO}_{3}$ increasing, $\mathrm{Ag}$ shell gradually became thickness. As is shown in Fig. 4, $\mathrm{UC} @ \mathrm{SiO}_{2} @ A u @ A g$ displayed stronger enhancement effect of

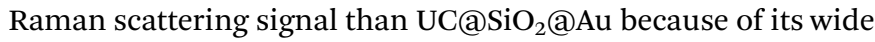
and strong plasmon resonance. Furthermore, Raman signal intensity of $1 \times 10^{3} \mathrm{mg} \mathrm{L}^{-1}$ HMT obviously increase with the volume of $\mathrm{AgNO}_{3}$ over $0.8-3.2 \mathrm{~mL}$ range, whereas a decrease is observed beyond $3.2 \mathrm{~mL}$. The results may be explained from its UV-vis spectra (shown in Fig. 5) that the increasing volume of $\mathrm{AgNO}_{3}$ resulted in blue-shifted of Au core resonance and a new resonance starts to be observed as well. When Ag shell resonance started to become slightly dominant, the strongest plasmon coupling between the Au core and $\mathrm{Ag}$ shell appeared. But with the further increase of $\mathrm{AgNO}_{3}, \mathrm{Ag}$ shell resonance started to become dominant and exhibited a greater blue-shift of plasmon coupling result in Raman signal intensity decreasing. ${ }^{23,24}$ So $3.2 \mathrm{~mL} \mathrm{AgNO}_{3}$ was selected in the synthetic experiments.

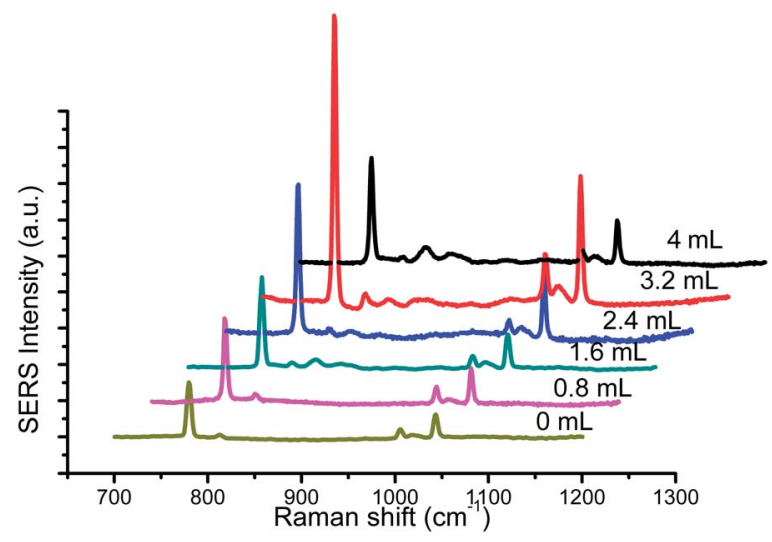

Fig. 4 The Raman spectra of $1 \times 10^{3} \mathrm{mg} \mathrm{L}^{-1} \mathrm{HMT}$ with different volume of $\mathrm{AgNO}_{3}(0,0.8,1.6,2.4,3.2,4 \mathrm{~mL}), 40 \mu \mathrm{L}$ TEOS, $100 \mu \mathrm{L}$ Au@Ag NPs.

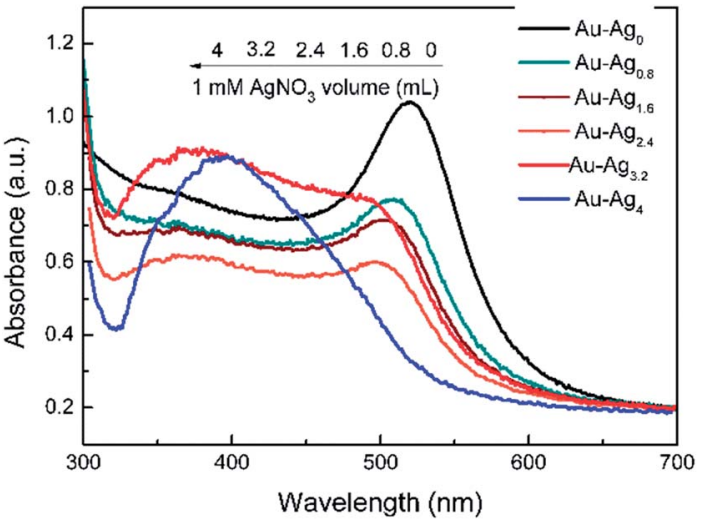

Fig. 5 The UV-vis spectra of Au@Ag with different amounts of $\mathrm{AgNO}_{3}$.

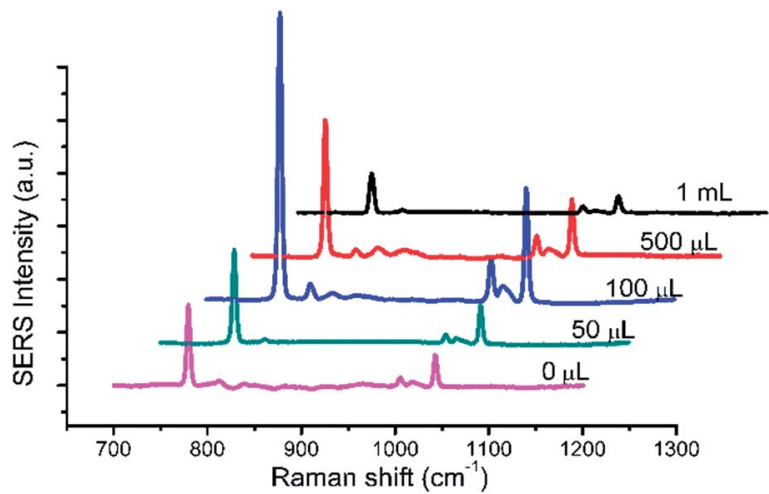

Fig. 6 The Raman spectra of $1 \times 10^{3} \mathrm{mg} \mathrm{L}^{-1} \mathrm{HMT}$ with different amounts of Au@Ag NPs. $40 \mu \mathrm{L}$ TEOS, $3.2 \mathrm{~mL} \mathrm{AgNO}_{3}$

The effect of different volume of Au@Ag NPs must be taken into account because Au@Ag NPs is a critical element to form hot spots to improve Raman signal intensity. The results were presented in Fig. 6. With the increase of volume of Au@Ag NPs, the Raman signal intensity was obviously increased with the amount from $50 \mu \mathrm{L}$ to $100 \mu \mathrm{L}$, whereas the increase was gradual decrease over $100 \mu \mathrm{L}$. Due to the increasing amount from $50 \mu \mathrm{L}$ to $100 \mu \mathrm{L}$, the more amount of Au@Ag NPs is easier to form hot spot to cause a boost of the Raman intensity. However, the excess amount of Au@Ag NPs may influence the upconversion efficiency to lead to decrease of Raman intensity.

\subsection{The uniformity of Raman substrate}

It is necessary to verify the uniformity of substrate under the optimized synthetic conditions. Raman spectra of $1 \times$ $10^{3} \mathrm{mg} \mathrm{L}^{-1}$ HMT were collected. The intensity of the main vibration of HMT from 20 SERS data was showed in Fig. 7. The obvious bands of HMT can be observed at $\sim 780 \mathrm{~cm}^{-1}$ and $\sim 1043 \mathrm{~cm}^{-1}$. All the selected Raman spectra were placed in a same horizontal line ( $y$-axis is about 0). Fig. 7 showed the relative standard deviation (RSD) of the Raman vibrations at $\sim 780 \mathrm{~cm}^{-1}$ and $\sim 1043 \mathrm{~cm}^{-1}$ were $7.05 \%$ and $7.37 \%$, respectively, which evidently revealing the high uniform of the Raman substrate. As we all know, generating good SERS performance is 

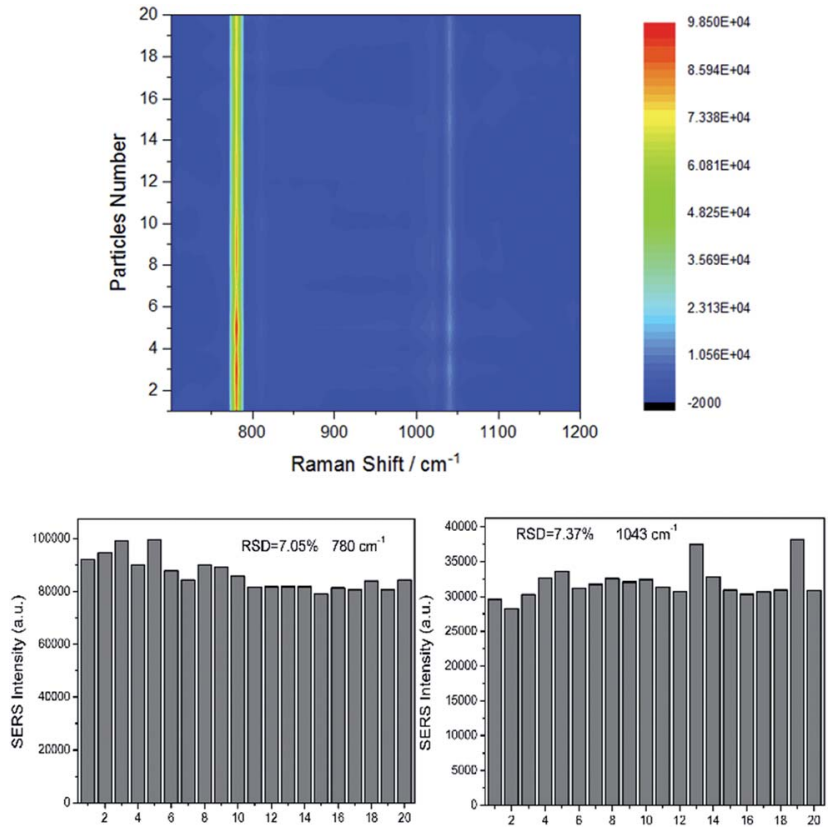

Fig. 7 The intensity of the main vibration of HMT from 20 SERS data, RSD of the Raman vibrations at $\sim 780 \mathrm{~cm}^{-1}$ and the Raman vibrations at $\sim 1043 \mathrm{~cm}^{-1}$.

related to LSPR of noble metal nanoparticles. ${ }^{7,24}$ Actually, the LSPR frequency of Au@Ag usually locate in the visible range, in other words visible light can easily match LSPR frequency of $\mathrm{Au}$ @Ag. ${ }^{14}$ In the $\mathrm{NaYF}_{4}, \mathrm{Yb}$, Er structure, the function of $\mathrm{Er}^{3+}$ is converting NIR or IR to visible light. The addition of $\mathrm{Yb}^{3+}$, as sensitizers, can transfer energy from $\mathrm{Yb}^{3+}$ to $\mathrm{Er}^{3+}$ to enhance the luminous efficiency. ${ }^{25}$ Under the NIR excitation of $785 \mathrm{~nm}$, the UC@SiO $\mathrm{S}_{2} @ \mathrm{Au} @ A g$ can boost Raman signals.

\subsection{The influences of detection conditions on Raman spectra intensity}

The influences of the amount of the UC@SiO added parts of the UC@SiO $\mathrm{U}_{2} @ \mathrm{Au} @ A g$ on Raman spectra intensity were taken into account.

The spectra of $2.5 \mu \mathrm{L} 1 \times 10^{3} \mathrm{mg} \mathrm{L}^{-1}$ HMT with the amount of $\mathrm{UC} @ \mathrm{SiO}_{2} @ \mathrm{Au} @ \mathrm{Ag}$ from $1 \mu \mathrm{L}$ to $4 \mu \mathrm{L}$ added by once were

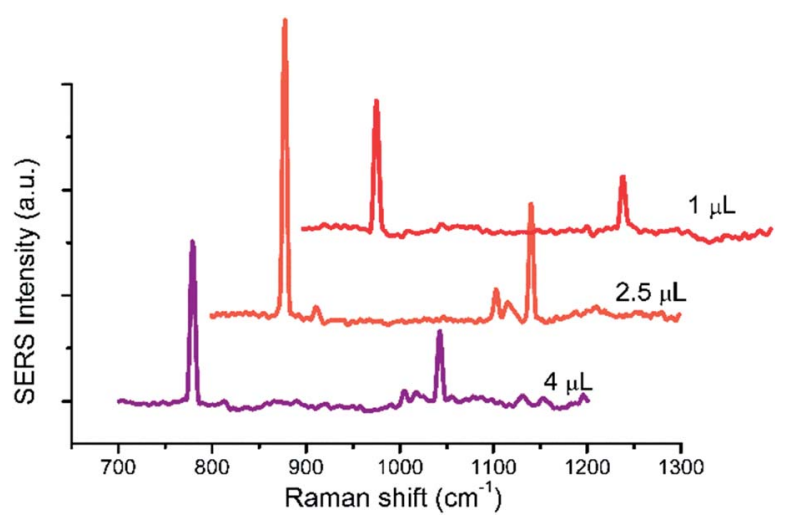

Fig. 8 The spectra of $2.5 \mu \mathrm{L} 1 \times 10^{3} \mathrm{mg} \mathrm{L}^{-1} \mathrm{HMT}$ with the amount of UC@SiO 2 @Au@Ag from $1 \mu \mathrm{L}$ to $4 \mu \mathrm{L}$.

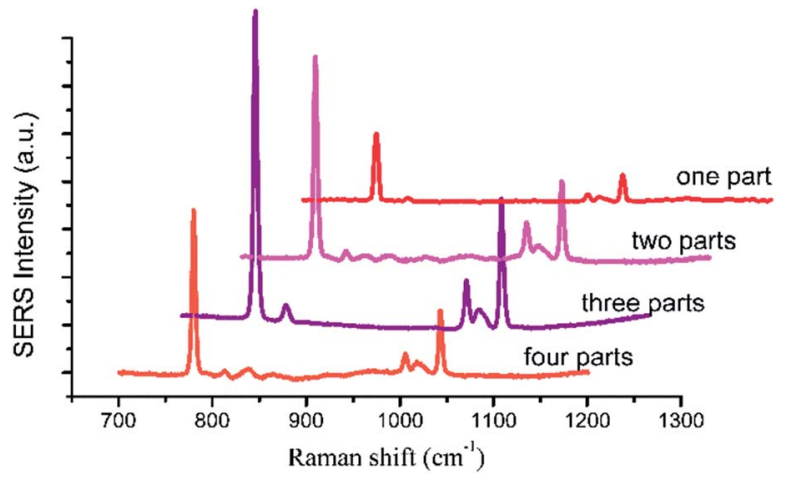

Fig. 9 The spectra of $2.5 \mu \mathrm{L} 1 \times 10^{3} \mathrm{mg} \mathrm{L}^{-1}$ HMT with the $\mathrm{UC} \mathrm{aSiO}_{2} \mathrm{aAu}$ aAg added-parts from one to four parts.

recorded (Fig. 8). The Raman intensity of HMT showed obvious increase with the amount from $1 \mu \mathrm{L}$ to $2.5 \mu \mathrm{L}$, whereas the Raman intensity decreased beyond $2.5 \mu \mathrm{L}$. Due to the more UC@SiO $\mathrm{S}_{2} @ \mathrm{Au} @ A g$ were aggregated on the plate with the increasing amount of $\mathrm{UC} @ \mathrm{SiO}_{2} @ \mathrm{Au} @ \mathrm{Ag}$, it cause a boost of the Raman intensity. However, when UC@SiO $\mathrm{U}_{2} @ \mathrm{Au} @ A g$ were aggregated too much to result in the Raman intensity decreasing.

The spectra of $2.5 \mu \mathrm{L} 1 \times 103 \mathrm{mg} \mathrm{L}^{-1}$ HMT with the $\mathrm{UC} @ \mathrm{SiO}_{2} @ \mathrm{Au} @ \mathrm{Ag}$ added-parts from one to four parts were collected (Fig. 9). As shown in Fig. 9, when $2.5 \mu \mathrm{L}$ of $\mathrm{UC} @ \mathrm{SiO}_{2} @ \mathrm{Au} @ A \mathrm{Ag}$ were divided into several parts from one to three added to the plate, the SERS signal of the HMT became stronger. When the added parts over three parts, the Raman intensity of HMT decreased. On the one hand, this may be because the UC@SiO $\mathrm{SiAu}_{2} @ \mathrm{Ag} w \mathrm{w}$ diffused to the edge to lead to fewer UC@SiO

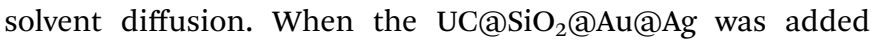
repeatedly, the $\mathrm{UC} @ \mathrm{SiO}_{2} @ A u @ A g$ was gradually form monolayer coverage to make the Raman intensity of HMT increased. When the added parts over three parts, the UC@SiO ${ }_{2} @ A u @ A g$ form multilayer accumulation and the SERS signal will be reduced accordingly. On the other hand, with added-parts increasing, more particle-particle interactions encouraged more UC@SiO $\mathrm{S}_{2} @ \mathrm{Au} @ \mathrm{Ag}$ to be aggregated and appropriate

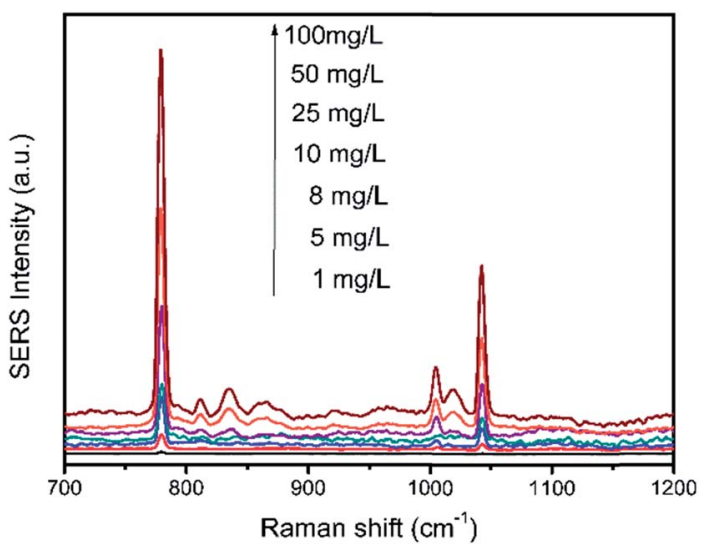

Fig. 10 The SERS spectra of different concentrations from $1 \mathrm{mg} \mathrm{L}^{-1}$ to $100 \mathrm{mg} \mathrm{L}^{-1}$. 


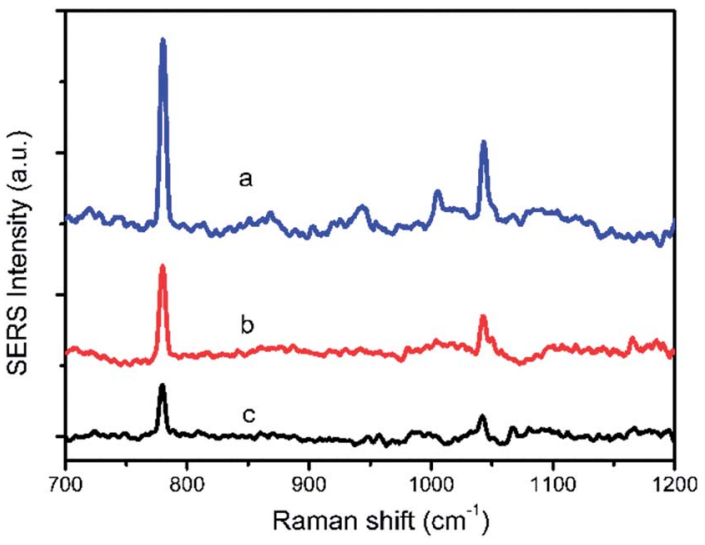

Fig. 11 The SERS spectra of artificially positive samples from different concentrations, $80 \mathrm{mg} \mathrm{L}^{-1}$ soaking concentrations (a) $50 \mathrm{mg} \mathrm{L}^{-1}$ soaking concentrations (b) $20 \mathrm{mg} \mathrm{L}^{-1}$ soaking concentrations (c).

aggregation caused a boost of the SERS intensity, but excessive aggregation reduced the SERS intensity.

\subsection{Sensitivity and practicability of detection method}

$2 \mu \mathrm{L}$ of HMT solution were dropped to $2.5 \mu \mathrm{L}$ of substrate and the Raman spectra of different concentrations from $1 \mathrm{mg} \mathrm{L}^{-1}$ to $100 \mathrm{mg} \mathrm{L}^{-1}$ were shown in Fig. 10. It can be seen that, the SERS signals decrease apparently with the reduction in HMT concentration. However, in a lower concentration of $1 \mathrm{mg} \mathrm{L}^{-1}$, the main characteristic peaks of HMT were still clearly observed. The LOD of the HMT was determined to be $1 \mathrm{mg} \mathrm{L}^{-1}$.

Bean vermicelli was chose as real sample to demonstrate the applicability of the detection method. The results indicated that no specific signal of HMT was observed in the bean vermicelli sample detected by the proposed method and HPLC, which meant that there was no HMT or the concentration of HMT residual in the bean vermicelli was below the detection limit. So the three kinds of the artificially positive samples were prepared by soaking bean vermicelli into different concentrations of HMT solution and detected by the proposed method under the optimal conditions. The results showed that HMT was remained on the bean vermicelli by soaking bean vermicelli and whether in low or high soaking concentrations, this method can both detect successfully (Fig. 11). Furthermore, this method does not require any complicated sample pre-treatment and only require a minute amount of sample for testing. So this method was feasible in real samples detection.

\section{Conclusions}

In summary, a SERS substrate with UC assembling with Au@Ag has been prepared. The preparation of UC@Au@Ag is easily carried out by simple mixing. The experiment results demonstrate the substrate can be successfully applied to detect HMT in real samples (artificially positive samples) by simple pretreatment. The proposed method may be a promising analytical method for rapid and easy detection illegal addition in food products.

\section{Conflicts of interest}

There are no conflicts to declare.

\section{Acknowledgements}

This work was supported by the Ministry of Science and Technology of China (No. 2012AA101602 and No. 2012BAK17B03), and the National Key Research and Development Program of China (No. 2016YFD0401204).

\section{Notes and references}

1 E. Ariëns, A. Hanselaar, P. T. Henderson and A. Simonis, Eur. J. Clin. Pharmacol., 1982, 23, 373-375.

2 H. S. Lim, J. Y. Hwang, J. I. Kim, J. C. Choi and M. Kim, Food Sci. Biotechnol., 2016, 25, 1305-1311.

3 X. Xu, X. Zhang, S. Abbas, E. Karangwa, E. Duhoranimana, F. Peng and P. Shu, Food Control, 2015, 57, 89-95.

4 X. Xu, X. Zhang, E. Duhoranimana, Y. Zhang and P. Shu, Food Control, 2016, 61, 99-104.

5 X. Hu, G. Fang, A. Han, Y. Fu, R. Tong and S. Wang, J. Sep. Sci., 2017, 40, 2506-2514.

6 J. Feng, L. Xu, G. Cui, X. Wu, W. Ma, H. Kuang and C. Xu, Biosens. Bioelectron., 2016, 81, 138-142.

7 P. L. Stiles, J. A. Dieringer, N. C. Shah and R. P. Van Duyne, Annu. Rev. Anal. Chem., 2008, 1, 601-626.

8 C. S. Ah, S. D. Hong and D.-J. Jang, J. Phys. Chem. B, 2001, 105, 7871-7873.

9 J. Long, H. Yi, H. Li, Z. Lei and T. Yang, Sci. Rep., 2016, 6, 33218.

10 Z. Yi, G. Niu, J. Luo, X. Kang, W. Yao, W. Zhang, Y. Yi, Y. Yi, X. Ye, T. Duan and Y. Tang, Sci. Rep., 2016, 6, 32314.

11 Z. Chen, H. Chen, H. Hu, M. Yu, F. Li, Q. Zhang, Z. Zhou, T. Yi and C. Huang, J. Am. Chem. Soc., 2008, 130, 30233029.

12 K. Qian, G. Fang and S. Wang, RSC Adv., 2013, 3, 3825.

13 A. Qu, X. Wu, L. Xu, L. Liu, W. Ma, H. Kuang and C. Xu, Nanoscale, 2017, 9, 3865-3872.

14 Y. Ma, H. Liu, Z. Han, L. Yang and J. Liu, Analyst, 2015, 140, 5268-5275.

15 Y. Ma, H. Liu, Z. Han, L. Yang, B. Sun and J. Liu, Analyst, 2014, 139, 5983-5988.

16 J. Wang, R. Liu, C. Zhang, G. Han, J. Zhao, B. Liu, C. Jiang and Z. Zhang, RSC Adv., 2015, 5, 86803-86810.

17 X. Ji, X. Song, J. Li, Y. Bai, W. Yang and X. Peng, J. Am. Chem. Soc., 2007, 129, 13939-13948.

18 A. Li, L. Tang, D. Song, S. Song, W. Ma, L. Xu, H. Kuang, X. Wu, L. Liu, X. Chen and C. Xu, Nanoscale, 2016, 8, 1873-1878.

19 X. Wu, X. Chen, F. Gao, W. Ma, L. Xu, H. Kuang, A. Li and C. Xu, Biosens. Bioelectron., 2016, 75, 55-58.

20 L. Wang and Y. Li, Chem. Mater., 2007, 19, 727-734.

21 E. Palo, L. Pihlgren, M. Tuomisto, T. Laihinen, I. Hyppänen, J. Kankare, M. Lastusaari, T. Soukka, H. C. Swart and J. Hölsä, Opt. Mater., 2016, 59, 49-54. 
22 P. Zhao, Y. Zhu, X. Yang, X. Jiang, J. Shen and C. Li, J. Mater. Chem. A, 2014, 2, 16523-16530.

23 B. Liu, G. Han, Z. Zhang, R. Liu, C. Jiang, S. Wang and M. Y. Han, Anal. Chem., 2012, 84, 255-261.
24 J.-F. Li, Y.-J. Zhang, S.-Y. Ding, R. Panneerselvam and Z.-Q. Tian, Chem. Rev., 2017, 117, 5002-5069.

25 Y. Sun, Y. Chen, L. Tian, Y. Yu, X. Kong, J. Zhao and H. Zhang, Nanotechnology, 2007, 18, 275609. 